\title{
Aquapuncture Using Stem Cell Therapy to Treat Mdx Mice
}

\author{
Greyson Vitor Zanatta Esper, ${ }^{1}$ Graciela Conceição Pignatari, ${ }^{1,2}$ \\ Marcio Nogueira Rodrigues, ${ }^{1}$ Heloisa Godoi Bertagnon, ${ }^{3}$ Isabella Rodrigues Fernandes, ${ }^{1,2}$ \\ Nanci Nascimento, ${ }^{4}$ Angela Maria Florencio Tabosa, ${ }^{5}$ \\ Patrícia Cristina Baleeiro Beltrão-Braga, ${ }^{1,2,6}$ and Maria Angelica Miglino ${ }^{1,2}$
}

\author{
${ }^{1}$ Stem Cell Lab, Surgery Department, School of Veterinary Medicine, University of São Paulo, 87 Prof. Dr. Orlando Marques de Paiva \\ Avenue, 05508-270 São Paulo, SP, Brazil \\ ${ }^{2}$ Center for Cellular and Molecular Studies and Therapy (NETCEM), São Paulo, SP, Brazil \\ ${ }^{3}$ Clinic Department, School of Veterinary Medicine, University of São Paulo, 87 Prof. Dr. Orlando Marques de Paiva Avenue, \\ 05508-270 São Paulo, SP, Brazil \\ ${ }^{4}$ Nuclear and Energy Research Institute Biotechnology Center, 2242 Prof. Lineu Prestes Avenue, 05508-270 São Paulo, SP, Brazil \\ ${ }^{5}$ Division of Chinese Medicine and Acupuncture, Federal University of São Paulo, 862 Botucatu Street, \\ 04023-900 São Paulo, SP, Brazil \\ ${ }^{6}$ Obstetrics Department, School of Arts, Sciences and Humanities, University of São Paulo, 1000 Arlindo Bettio Avenue, \\ 03828-000 São Paulo, SP, Brazil
}

Correspondence should be addressed to Graciela Conceição Pignatari; grapignatari@yahoo.com.br

Received 2 March 2015; Accepted 19 April 2015

Academic Editor: Andreas Sandner-Kiesling

Copyright (C) 2015 Greyson Vitor Zanatta Esper et al. This is an open access article distributed under the Creative Commons Attribution License, which permits unrestricted use, distribution, and reproduction in any medium, provided the original work is properly cited.

\begin{abstract}
Duchenne muscular dystrophy (DMD) occurs due to genetic mutations that lead to absence or decrease of dystrophin protein generating progressive muscle degeneration. Cell therapy using mesenchymal stem cell (MSC) has been described as a treatment to DMD. In this work, MSC derived from deciduous teeth, called stem cells from human exfoliated deciduous teeth (SHED), were injected in acupoint as an alternative therapy to minimize muscle degeneration in twenty-two mdx mice. The treatment occurred three times with intervals of 21 days, and animals were analyzed four times: seven days prior treatment (T-7); 10 days after first treatment (T10); 10 days after second treatment (T31); and 10 days after third treatment (T52). Animals were evaluated by wire test for estimate strength and blood was collected to perform a creatinine phosphokinase analysis. After euthanasia, cranial tibial muscles were collected and submitted to histological and immunohistochemistry analyses. Treated groups presented improvement of strength and reduced creatinine phosphokinase levels. Also, a slight dystrophin increase was observed in tibial cranial muscle when aquapuncture was associated SHED. All therapies have minimized muscle degeneration, but the association of aquapuncture with SHED appears to have better effect, reducing muscle damage, suggesting a therapeutic value.
\end{abstract}

\section{Introduction}

Muscular dystrophies are neuromuscular diseases genetically related that compromise progressively skeletal muscles. Regarding that, Duchenne muscular dystrophy (DMD) presents worldwide frequency of approximately 1:3500 male children. DMD is a recessive inheritance pattern, with the first symptoms starting at 3-6 years old [1-3]. The DMD mutation occurs in a gene located on the $\mathrm{X}$ chromosome
(Xp21), responsible for encoding dystrophin $[1,4]$, a structural protein that acts stabilizing the sarcolemma muscle, connecting the actin cytoskeleton with the extracellular matrix [5]. Dystrophin deficiency affects the contraction physiology, causes sarcolemma injury, and leads to cell death [6].

Several studies tried some therapeutic strategies to treat DMD, such as antibiotics, glucocorticoids, and calcium channel blockers [7-11]. Cell therapy using stem cells has 
also been tested $[9,11-15]$. Besides stem cell hypothetically could replace damaged cell, and stem cells also are able to perform immunomodulation activities that could balance the progression of muscular dystrophy by anti-inflammatory effect [7, 14, 16-18]. In addition, gene therapy has been tested to treat DMD [19-22] and, recently, a combination of stem cells, using induced pluripotent stem cells (iPSC) from a patient with DMD, and gene therapy, correcting genetically the cells using TALENs or CRISPR, restored dystrophin production in vitro [22].

Stem cells from human exfoliated deciduous teeth (SHED) were first isolated as a population of multipotent stem cells from the pulp of exfoliated deciduous teeth [23]. SHED are able to differentiate into odontogenic, adipogenic, osteogenic, neural, hepatocytes, and endothelial cells [24$27]$, have immunomodulatory properties $[27,28]$, and are able to perform cell reprogramming [29]. Also, stem cells from dental pulp were used to treat Golden Retriever muscular dystrophy dogs providing some dystrophin expression [30].

The dystrophin deficient mice, mdx (C57BL10 ScSnDMD-mdx/J) are widely used as an animal model to study DMD in order to investigate various aspects of this disease and for preclinical test or proof-of-concept studies. Mdx mice have a milder clinical behavior than human, presenting muscle degeneration in waves leading to necrosis, as well as presenting some serum biochemical parameters and histological changes, similar to human affected by DMD [31, 32].

Acupuncture, a traditional oriental medicine is therapeutic approach that has been used to contribute to pain control, analgesia, inflammatory process, and others factors improving the treatment in several degenerative diseases like Parkinson's, DMD, and spinal cord injury [33-36]. Aquapuncture is a variation of acupuncture that involves the application of aqueous substances (like drugs) in the acupoints in order to potentiate therapeutic action [37]. This technique combines acupoints stimulation and the pharmacological substances improving beneficial effect if used separately [37]. The association of acupuncture with cell therapy is new, but some studies in this direction have already been performed. Recently, acupoints injection associated with mesenchymal stem cell (MSC) was used to treat hip dysplasia in dogs presenting positive results, showing increased functional improvement [38].

Regarding this possible beneficial effect, this study aimed to use acupoint injection with SHED in order to verify the benefits of this approach to treat $\mathrm{mdx}$ mice.

\section{Materials and Methods}

2.1. Animals. This work was approved by the ethics committee on animal use, from the School of Veterinary Medicine and Animal Science, São Paulo University, protocol number 2431/2011. Animal research was conducted in accordance with the Ethical Principles in Animal Experimentation, according to the National Institute of Health Guide for the Care and Use of Laboratory Animals, 1996. Twenty-two male mdx mice (14-20 g) between 4 and 6 weeks of age from IPEN animal facility (Nuclear and Energy Research Institute, Brazil) were used in this work. Animals were kept in cage $(40 \times 34 \times 17 \mathrm{~cm})$ with wood shavings, light/dark cycle $(12 / 12 \mathrm{~h})$ in controlled temperature $\left(20 \pm 1^{\circ} \mathrm{C}\right)$ and humidity $(50 \pm 10 \%)$, feed (Nuvital, PR, Brazil), and water ad libitum. The maximum number of animals per cage was 5, divided according with treatment received.

2.2. SHED. SHED were obtained using a modified Miura's protocol [12], with collagenase type I for pulp digestion $(3 \mathrm{mg} / \mathrm{mL})$. SHED were carefully prepared to animal infusion. After trypsinization, cells were washed twice and counted and $1 \times 10^{4}$ cells were ressuspended in saline solution (Sigma-Aldrich, MO, USA) in a very low volume (20 microliters).

2.3. Acupoint Associated with Stem Cell Injection. Four groups of animals were distributed to do the following treatments: (A) SHED injection in false acupoint $(n=5)$; (B) saline injection in true acupoint $(n=5)$; (C) SHED in true acupoint $(n=5)$; and (D) control, without treatment $(n=$ 5). SHED were injected in each acupoint every three weeks (21 days of interval each), totaling three injections during the whole experiment. Acupoints selected for injection were Bladder 47 (Hunmen), Bladder 49 (Yishe), and Bladder 52 (Zhishi) (Figure 1(a)), selected based on Traditional Chinese Medicine because these acupoints can connect the meridian and could improve the effect in the body. After treatments mdx were analyzed at four moments: (T-7) seven days before treatment; (T10) 10 days after the first SHED transplantation; (T31) 10 days after the second SHED transplantation; and (T52) 10 days after the third SHED cell transplantation, according to the timeline (Figure 1(b)). Before the injection mice used chemical restraint and inhalational anesthesia in an open system with isoflurane 3\% (Cristália, SP, Brazil). Placebo groups were injected with 20 microliters of saline solution at same acupoints and conditions.

2.4. Acupoint Treatment Analyses. Animals were submitted to clinical evaluation by wire test, creatinine phosphokinase, and histological analyses.

2.4.1. Wire Test. The strength of the animal's forelimbs was assessed by wire test adapted from van Putten et al. (2012) [6]. For this test, mice were suspended on a metal cloth hanger secured $35 \mathrm{~cm}$ above the cage. Animals were analyzed until the fall, for 4 consecutive times, all recorded by video. The forelimbs strength was quantified using a rating scale considering the following parameters: (1) raise the body above the wire, (2) walk on the wire, (3) put the pelvic member on the wire; and (4) time on the wire. Each parameter was analyzed by three double-blind raters (Table 1). After this evaluation the values obtained in Table 1 were summed for each animal and the animals were classified in poor, median, and strong according to muscle strength score. Results were plotted in GraphPad Prism 5 and analyzed using 2-way ANOVA. 
TABLE 1: Wire test score and the strength quantification. This table reveals the score attributed for each action using wire test. The total time used in this evaluation was 21 seconds. The number of times walking or holding on the wire was attributed considering 0 to 4 steps. For each animal, the points obtained from Table 1 were summed and according to the score obtained the animal was classified in poor ( $0-2$ points), median (3-5 points), and strong (6-8 points).

\begin{tabular}{lccc}
\hline Action & 0 step/holding until $9 \mathrm{~s}$ on the wire & 1 to 3 steps/holding $10-20 \mathrm{~s}$ on the wire & 4 or $>$ turn/21s \\
\hline Raise the body above the wire & 0 point & 1 point & 2 points \\
Walk on the wire & 0 point & 1 point & 2 points \\
Put the pelvic member on the wire & 0 point & 1 point & 2 points \\
Time on the wire (seconds only) & 0 point & 1 point & 2 points \\
\hline
\end{tabular}

2.4.2. Analysis of Serum Creatinine Phosphokinase (CPK). Animals' blood was collected in the facial vein and plasma was diluted $1: 100$. The samples were analyzed kinetically by CK-NAC kit (Randox Laboratories, London, England) in Biotek Powerwave XS spectrophotometer by the KC4 software (v. 3.4) (Biotek, CA, USA). Results were plotted in GraphPad Prism 5 and analyzed using 2-way ANOVA.

2.4.3. Histological Analysis. After animal's euthanasia, cranial tibial muscle and diaphragm were collected, frozen in liquid nitrogen, fixed in $4 \%$ paraformaldehyde, and subjected to routine histological procedures. Sections of $5 \mu \mathrm{m}$ thickness were performed in a microtome (Leica RM-2065, Germany) and stained with hematoxylin-eosin (HE) and visualized by light microscopy (Olympus BX60 microscope, Tokyo, Japan) coupled with an AxioCam (Zeiss, Jena, Germany). Immunofluorescence analyses on tibial cranial and diaphragm muscles sections were performed to analyze the presence of dystrophin, using serial sections of $10 \mu \mathrm{m}$ thickness accommodated on silanized slides. C57/BL6, animal without dystrophin pathology, was used here as a positive control for dystrophin expression. The samples were fixed with $100 \%$ acetone for 20 minutes at $-20^{\circ} \mathrm{C}$. Then, the slides were washed with PBS 3 times for $5 \mathrm{~min}$ each and added anti-dystrophin antibody (ab3149, Abcam, Cambridge, UK) diluted $(1: 100)$ in PBS containing $1 \%$ bovine serum albumin (BSA) in a humid chamber overnight at $4^{\circ} \mathrm{C}$. After this time, the samples were washed with PBS 3 times, 5 min each, and added secondary anti-rabbit IgG NL557 antibody (R\&D Systems, Minneapolis, USA) diluted 1:200 in PBS containing $1 \%$ BSA, kept in a dark chamber for 60 minutes at room temperature. Then, the slides were washed with PBS 5 times for 5 minutes each and mounted using Vectashield with DAPI (Vector Laboratories, Burlingame, CA, USA).

\section{Results and Discussion}

Here we proposed an alternative method to treat DMD using mdx as a model to test the benefits of acupuncture associated with stem cell therapy, a pioneer work in this field. Acupoints were selected bilaterally after an accurate assessment of DMD, associated with the benefits they could bring from Traditional Chinese Medicine. So, in this project the points used to apply acupuncture were Bladder 47 (B47), Bladder 49 (B49), and Bladder 52 (B52) based on the fact that DMD patients present a deficiency in liver, spleen-pancreas, and kidney meridians and the points chosen could nourish the first column of Shu

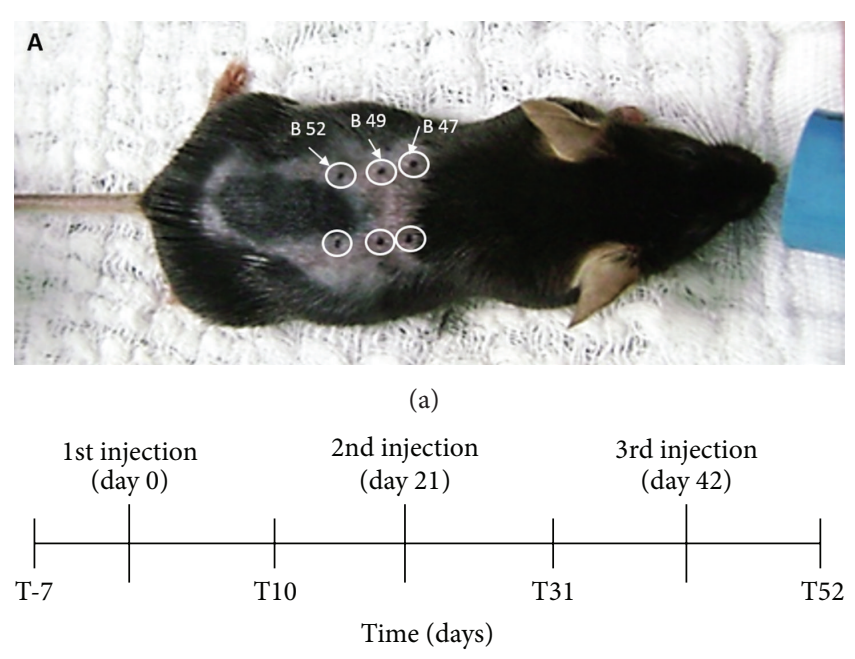

(b)

Figure 1: Acupoint injections and timeline of treatments. (a) Acupoints used in this study in mdx mice: BL47, BL49, and BL52 (white arrows). (b) Experimental timeline considering days of injection and days for treatment evaluation. (T-7) seven days before treatment; (T10) ten days after first treatment (SHED or saline injection); (T31) twenty-one days after second treatment (SHED or saline injection); (T52) twenty-one days after third treatment (SHED or saline injection).

points [39]. The B47 point is located on the side depression to the edge flow, related to the spinous process of the vertebra. The B49 point is located on the side depression of the spinous process of the twelfth vertebra and is related to the spinous process of the vertebra. The B52 point is located on the side depression of the spinous process of the second lumbar vertebra, which is also related to the spinous processes of the vertebrae (Figure 1(a)).

In this work, mdx mice were evaluated for the total time of 52 days (Figure 1(b)), which is considered enough time to check mdx muscle degeneration according to natural disease evolution in this animal model [40]. As here $\mathrm{mdx}$ mice remained less time on the wire and fatigue more than observed by van Putten's wire test [6]. For this reason the test was adapted including other parameters, such as erection of the body on the wire, walking on the wire, and placement of the pelvic limb on the wire. In addition, scales for scoring behaviors and the quality of strength, like weak, medium, and strong, were assigned (Table 1). 


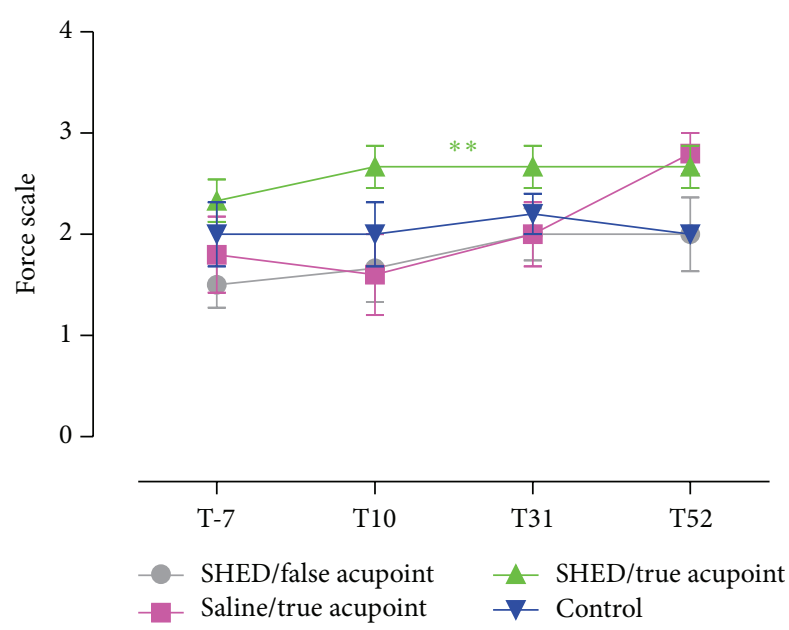

(a)

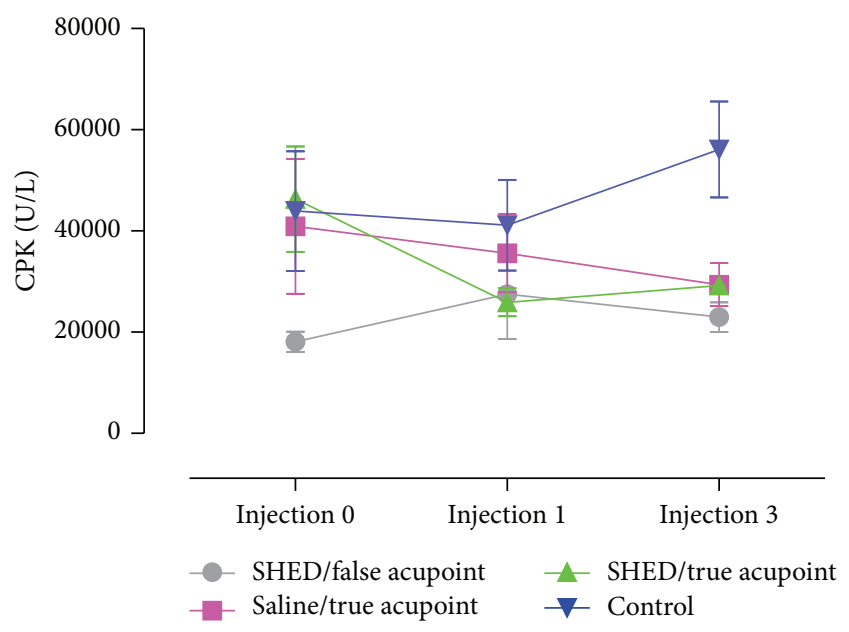

(b)

FIGURE 2: Analysis for the treatment using wire test and seric CPK, (a) scale force graphic using wire test, (b) seric CPK analysis. $* *$ means $p$ value $=0.0021$.

Concerning force using an adapted wire test, statistical differences were observed comparing treatments, with a strength improvement in animals submitted to SHED/true acupoints ( $p$ value: 0.0021 ) and a slight strength improvement in SHED/false acupoints and saline/true acupoints, always comparing with animal without any treatment, called controls (Figure 2(a)). However, the improvement of force in SHED true acupoint suggested clinical progress in muscle which is interesting making acupuncture an alternative therapy but not enough to revert the physiological behavior. Force improvement using just acupuncture was related before using the E36, BP6, and auricular Shenmen points in mdx showing a positive result [41], like observed here. Moreover, concerning stem cell treatment to $\mathrm{mdx}$, when adipose stem cells were injected intramuscularly in mdx mice increasing muscle strength and resistance to muscle fatigue were observed [39].

Serum CPK analysis is a reliable measurement for the identification and quantification of muscle damage. This analysis infers animals' improvement or worsening during the treatment [42-44]. Increasing of CPK suggests muscle damage during the peak of muscle degeneration in $\mathrm{mdx}$ mice, which occurs between 35 and 90 days of age [40]. Here, CPK decreased approximately 1.5 times less than the control using SHED/true acupoints, which suggest a clinical improvement in this animal group. Seric CPK revealed that all treated animals presented CPK decrease, although significant differences were not found (Figure 2(b)).

The causes of the perpetuation of muscle degeneration are numerous such as free radicals release and lack of the dystrophin $[45,46]$. Therefore, stem cells effect in false acupoints or placebos could result in the same mechanism of action, explaining the reduction in plasma CPK among treatments. The CPK decreasing is a good parameter to monitor the treatment $[47,48]$.

In addition, we observed a delay in disease progression in animals treated with SHED in false acupoints suggesting that cells could present a paracrine effect, which is already, known for SHED [27].

Histological analysis inferred that treatments did not interfere with anatomical structure of tissue and did not produce inflammatory reaction (Figures $3(\mathrm{a})-3(\mathrm{~d})$ ).

Immunohistochemistry using dystrophin protein analysis in cranial tibial muscle in $\mathrm{mdx}$ revealed that all treated groups presented a slight dystrophin improvement (Figures 3(e)-3(g)), comparing with the control (mdx without treatment Figure 3(h)), being more evident in SHED/true acupoint and SHED/false acupoint, suggesting cell influence. However, even getting a little better, visually, dystrophin amount was lower in mdx mice than in C57/BL6, animal without dystrophin pathology, used here as a positive control for dystrophin expression (Figure 3(i)). In spite of mdx mice treated with SHED in true acupoint presented an increase of dystrophin expression in tibial cranial muscle; this was not sufficient to improve muscle tissue normal appearance (Figure $3(\mathrm{~g})$ ), suggesting that regenerative ability might not be directly related to dystrophin presence. The increase of dystrophin production was also observed in mdx mice treated with some drugs, as gentamicin, pentoxifylline, and RTC 13 and 14 molecules [42, 43, 49] being an important improvement but without phenotype reversion. Just lately, genetic correction using CRISPR and TALEN was able to correct the dystrophin and permit normal gene expression of dystrophin [22].

Considering an acupuncture therapy for $\mathrm{mdx}$ and the results found here, acupuncture associated or not with SHED cells seems to increase dystrophin production and prevent the disease progression, being revealed as an alternative treatment for this untreated disease. An improvement in dystrophin expression was also observed in SHED/false acupoint suggesting the influence of cells in the treatment. Moreover, CPK decrease was observed in all treatments, but only SHED/true acupoint treatment was able to improve 


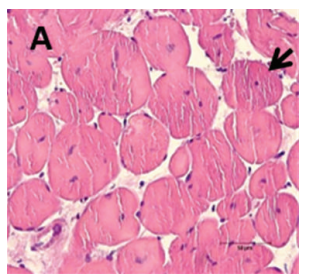

(a)

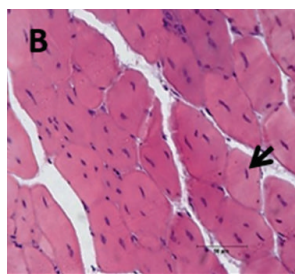

(b)

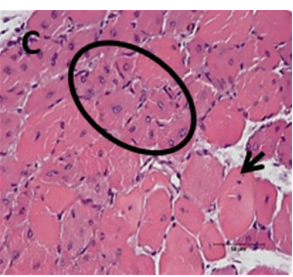

(c)

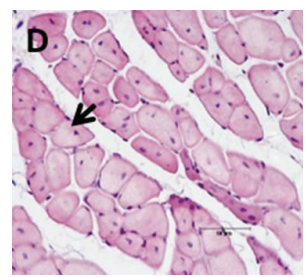

(d)
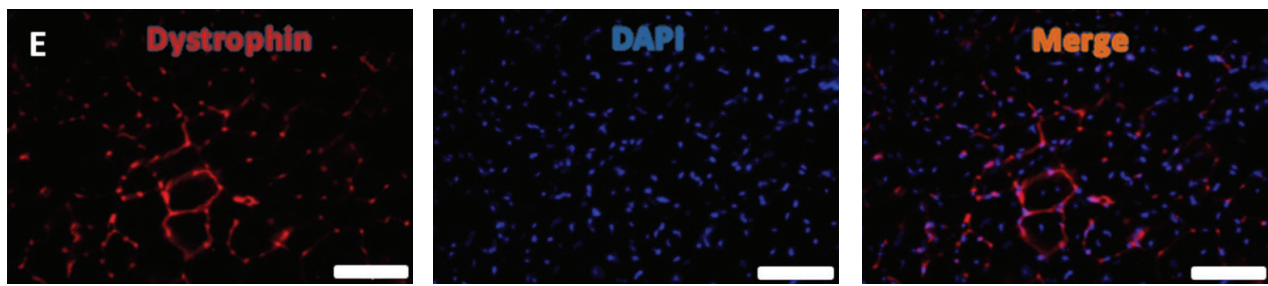

(e)
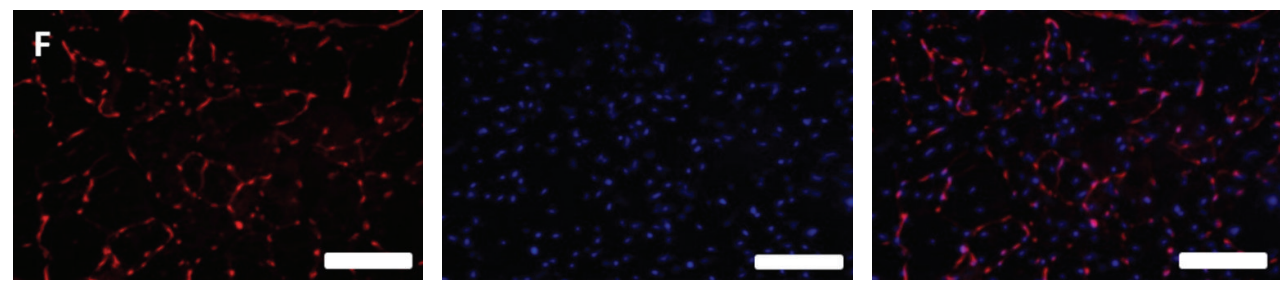

(f)
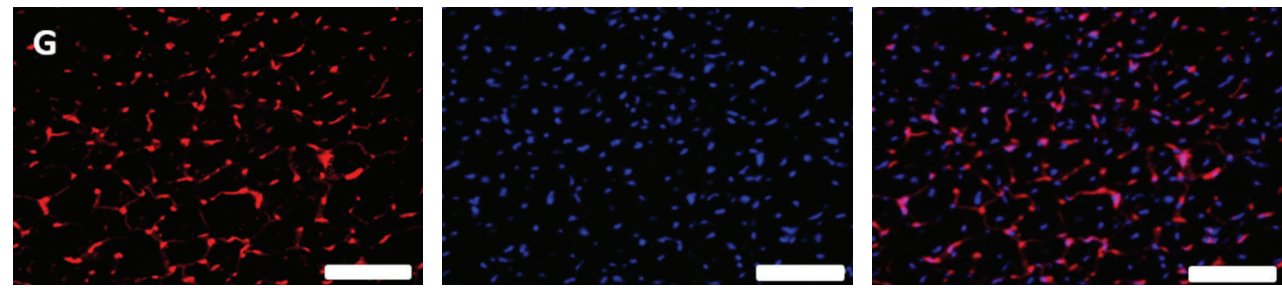

$(\mathrm{g})$
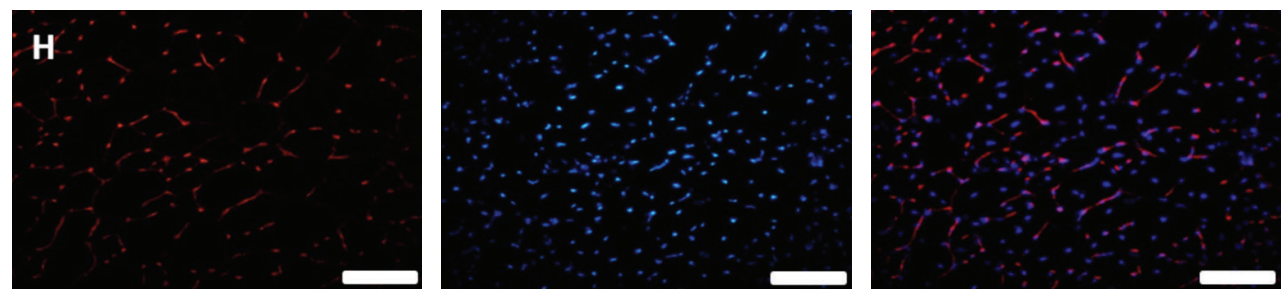

(h)
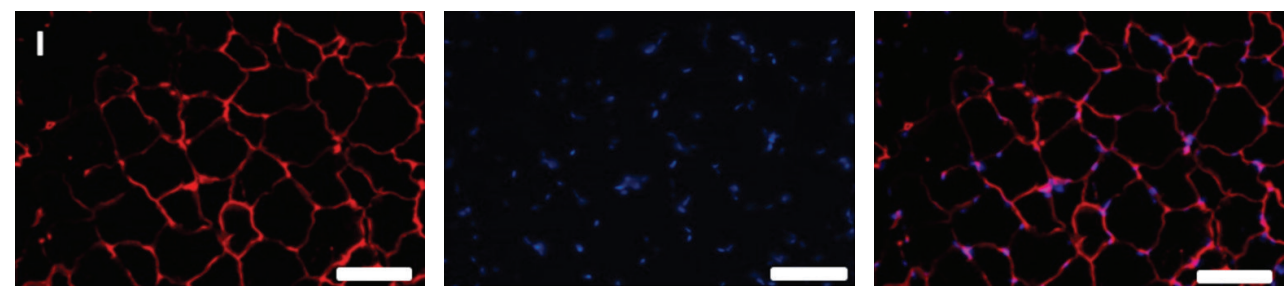

(i)

FIGURE 3: Histological analyses of cranial tibial muscle using HE staining (a-d). In (c), inside the circle, centralized nuclei and increased heterogeneity in myofibers size, characteristics of mdx muscle degeneration. Dystrophin expression in tibial cranial muscles after 52 days of the treatment (e-i). Groups: SHED/false acupoints; saline/true acupoints; SHED/true acupoints; and control. Scale Bar-100 $\mu \mathrm{m}$. 
force, an important clinical parameter to DMD patients. In this work we can infer that acupuncture associated with SHED has a beneficial effect in muscle force as well as dystrophin expression and CPK decrease.

\section{Conclusions}

The use of acupuncture associated with SHED therapy appears to have a benefic effect in reducing disease progression in mdx mice and so far open new perspectives and opportunities to DMD treatment or could, at least, improve the quality of patient's life.

\section{Conflict of Interests}

The authors declare that there is no conflict of interests regarding the publication of this paper.

\section{Authors' Contribution}

Greyson Vitor Zanatta Esper and Graciela Conceição Pignatari contributed equally and are both first authors and the same to Patricia Cristina Baleeiro Beltrão-Braga and Maria Angelica Miglino, who are both last authors.

\section{Acknowledgments}

The authors are thankful to the Institute for Energy and Nuclear Research for the support regarding the obtaining of animals specially Maria Neide F. Mascarenhas, Cecília S. Ferreira, and Ismária S. Reis. They are also thankful to the Department of Surgery, Anatomy of Wild and Domestic Animals Service and National Council of Technological and Scientific Development (CNPq), and Sao Paulo Research Foundation (FAPESP) for the financial support.

\section{References}

[1] K. Bushby, R. Finkel, D. J. Birnkrant et al., "Diagnosis and management of Duchenne muscular dystrophy, part 1: diagnosis, and pharmacological and psychosocial management," The Lancet Neurology, vol. 9, no. 1, pp. 77-93, 2010.

[2] C. Pasternak, S. Wong, and E. L. Elson, "Mechanical function of dystrophin in muscle cells," Journal of Cell Biology, vol. 128, no. 3, pp. 355-361, 1995.

[3] D. Verhaert, K. Richards, J. A. Rafael-Fortney, and S. V. Raman, "Cardiac involvement in patients with muscular dystrophies: magnetic resonance imaging phenotype and genotypic considerations," Circulation: Cardiovascular Imaging, vol. 4, no. 1, pp. 67-76, 2011.

[4] R. González-Ramírez, S. L. Morales-Lázaro, V. Tapia-Ramírez, D. Mornet, and B. Cisneros, "Nuclear and nuclear envelope localization of dystrophin Dp71 and dystrophin-associated proteins (DAPs) in the $\mathrm{C}_{2} \mathrm{C}_{12}$ muscle cells: DAPs nuclear localization is modulated during myogenesis," Journal of Cellular Biochemistry, vol. 105, no. 3, pp. 735-745, 2008.

[5] M. Vainzof, L. U. Yamamoto, P. M. Kossugue et al., "Animal models help to decipher human neuromuscular disorders," Neuroscience, vol. 13, pp. 18-20, 2005.
[6] M. van Putten, M. Hulsker, V. D. Nadarajah et al., "The effects of low levels of dystrophin on mouse muscle function and pathology," PLoS ONE, vol. 7, no. 2, Article ID e31937, 2012.

[7] T. S. Khurana and K. E. Davies, "Pharmacological strategies for muscular dystrophy," vol. 2, no. 5, pp. 379-390, 2003.

[8] N. P. Whitehead, C. Pham, O. L. Gervasio, and D. G. Allen, “NAcetylcysteine ameliorates skeletal muscle pathophysiology in mdx mice," The Journal of Physiology, vol. 586, no. 7, pp. 20032014, 2008 .

[9] J. V. Chakkalakal, J. Thompson, R. J. Parks, and B. J. Jasmin, "Molecular, cellular, and pharmacological therapies for Duchenne/Becker muscular dystrophies," The FASEB Journal, vol. 19, no. 8, pp. 880-891, 2005.

[10] K. M. Flanigan, K. Campbell, L. Viollet et al., "Anti-dystrophin $\mathrm{T}$ cell responses in duchenne muscular dystrophy: prevalence and a glucocorticoid treatment effect," Human Gene Therapy, vol. 24, no. 9, pp. 797-806, 2013.

[11] M. F. Phillips and R. Quinlivan, "Calcium antagonists for Duchenne muscular dystrophy," Cochrane Database of Systematic Reviews, no. 4, Article ID CD004571, 2008.

[12] A. Sharma, H. Sane, P. Badhe et al., "A clinical study shows safety and efficacy of autologous bone marrow mononuclear cell therapy to improve quality of life in muscular dystrophy patients," Cell Transplantation, vol. 22, no. 1, pp. S127-S138, 2013.

[13] A. Sharma, H. Sane, A. Paranjape, K. Bhagawanani, N. Gokulchandran, and P. Badhe, "Autologous bone marrow mononuclear cell transplantation in duchenne muscular dystrophy-a case report," American Journal of Case Reports, vol. 15, pp. 128-134, 2014.

[14] M. Noviello, F. S. Tedesco, A. Bondanza et al., "Inflammation converts human mesoangioblasts into targets of alloreactive immune responses: implications for allogeneic cell therapy of DMD," Molecular Therapy, vol. 22, no. 7, pp. 1342-1352, 2014.

[15] R. Fujita, K. Tamai, E. Aikawa et al., "Endogenous mesenchymal stromal cells in bone marrow are required to preserve muscle function in $m d x$ mice," Stem Cells, vol. 33, no. 3, pp. 962-975, 2015.

[16] T. E. Ichim, D. T. Alexandrescu, F. Solano et al., "Mesenchymal stem cells as anti-inflammatories: implications for treatment of Duchenne muscular dystrophy," Cellular Immunology, vol. 260, no. 2, pp. 75-82, 2010.

[17] A. I. Caplan, "Why are MSCs therapeutic? New data: new insight," The Journal of Pathology, vol. 217, no. 2, pp. 318-324, 2009.

[18] L. da Silva Meirelles, A. M. Fontes, D. T. Covas, and A. I. Caplan, "Mechanisms involved in the therapeutic properties of mesenchymal stem cells," Cytokine and Growth Factor Reviews, vol. 20, no. 5-6, pp. 419-427, 2009.

[19] Q. L. Lu, G. E. Morris, S. D. Wilton et al., "Massive idiosyncratic exon skipping corrects the nonsense mutation in dystrophic mouse muscle and produces functional revertant fibers by clonal expansion," The Journal of Cell Biology, vol. 148, no. 5, pp. 985-996, 2000.

[20] Q. L. Lu, C. J. Mann, F. Lou et al., "Functional amounts of dystrophin produced by skipping the mutated exon in the $\mathrm{mdx}$ dystrophic mouse," Nature Medicine, vol. 9, no. 8, pp. 1009-1014, 2003.

[21] C. Long, J. R. McAnally, J. M. Shelton, A. A. Mireault, R. BasselDuby, and E. N. Olson, "Prevention of muscular dystrophy in mice by CRISPR/Cas9-mediated editing of germline DNA," Science, vol. 345, no. 6201, pp. 1184-1188, 2014. 
[22] H. L. Li, N. Fujimoto, N. Sasakawa et al., "Precise correction of the dystrophin gene in duchenne muscular dystrophy patient induced pluripotent stem cells by TALEN and CRISPR-Cas9," Stem Cell Reports, vol. 4, no. 1, pp. 143-154, 2015.

[23] M. Miura, S. Gronthos, M. Zhao et al., "SHED: stem cells from human exfoliated deciduous teeth," Proceedings of the National Academy of Sciences of the United States of America, vol. 100, no. 10, pp. 5807-5812, 2003.

[24] N. Ishkitiev, K. Yaegaki, B. Calenic et al., "Deciduous and permanent dental pulp mesenchymal cells acquire hepatic morphologic and functional features in vitro," Journal of Endodontics, vol. 36, no. 3, pp. 469-474, 2010.

[25] N. Ishkitiev, K. Yaegaki, T. Imai et al., "High-purity hepatic lineage differentiated from dental pulp stem cells in serum-free medium," Journal of Endodontics, vol. 38, no. 4, pp. 475-480, 2012.

[26] V. T. Sakai, Z. Zhang, Z. Dong et al., "SHED differentiate into functional odontoblasts and endothelium," Journal of Dental Research, vol. 89, no. 8, pp. 791-796, 2010.

[27] T. Yamaza, A. Kentaro, C. Chen et al., "Immunomodulatory properties of stem cells from human exfoliated deciduous teeth," Stem Cell Research and Therapy, vol. 1, no. 1, article 5, 2010.

[28] M. Roemeling-van Rhijn, M. Khairoun, S. S. Korevaar et al., "Human bone marrow- and adipose tissue-derived mesenchymal stromal cells are immunosuppressive in vitro and in a humanized allograft rejection model," Journal of Stem Cell Research \& Therapy, vol. 6, no. 1, Article ID 20780, 2013.

[29] P. C. B. Beltrão-Braga, G. C. Pignatari, P. C. Maiorka et al., "Feeder-free derivation of induced pluripotent stem cells from human immature dental pulp stem cells," Cell Transplantation, vol. 20, no. 11-12, pp. 1707-1719, 2011.

[30] I. Kerkis, C. E. Ambrosio, A. Kerkis et al., "Early transplantation of human immature dental pulp stem cells from baby teeth to golden retriever muscular dystrophy (GRMD) dogs: local or systemic?" Journal of Translational Medicine, vol. 6, article 35, 2008.

[31] R. Willmann, S. Possekel, J. Dubach-Powell, T. Meier, and M. A. Ruegg, "Mammalian animal models for Duchenne muscular dystrophy," Neuromuscular Disorders, vol. 19, no. 4, pp. 241-249, 2009.

[32] T. A. Partridge, "The mdx mouse model as a surrogate for Duchenne muscular dystrophy," The FEBS Journal, vol. 280, no. 17, pp. 4177-4186, 2013.

[33] D. C. Choi, J. Y. Lee, Y. J. Moon, S. W. Kim, T. H. Oh, and T. Y. Yune, "Acupuncture-mediated inhibition of inflammation facilitates significant functional recovery after spinal cordinjury," Neurobiology of Disease, vol. 39, no. 3, pp. 272-282, 2010.

[34] Y. Jiang, K. K. Kwong, and J. Liu, "Acupuncture in the treatment of Parkinson's disease," The North American Journal of Medicine and Science, vol. 2, pp. 32-34, 2009.

[35] J.-L. Yang, J. S. C. Chen, Y.-F. Yang et al., "Neuroprotection effects of retained acupuncture in neurotoxin-induced Parkinson's disease mice," Brain, Behavior, and Immunity, vol. 25, no. 7, pp. 1452-1459, 2011.

[36] J. A. Urtizberea, Q.-S. Fan, E. Vroom, D. Récan, and J.C. Kaplan, "Looking under every rock: duchenne muscular dystrophy and traditional Chinese medicine," Neuromuscular Disorders, vol. 13, no. 9, pp. 705-707, 2003.

[37] C.-Y. Chen, C.-N. Lin, R.-S. Chern, Y.-C. Tsai, Y.-H. Chang, and C.-H. Chien, "Neuronal activity stimulated by liquid substrates injection at zusanli (ST36) acupoint: the possible mechanism of aquapuncture," Evidence-Based Complementary and Alternative Medicine, vol. 2014, Article ID 627342, 7 pages, 2014.

[38] C. Marx, M. D. Silveira, I. Selbach et al., "Acupoint injection of autologous stromal vascular fraction and allogeneic adiposederived stem cells to treat hip dysplasia in dogs," Stem Cells International, vol. 2014, Article ID 391274, 6 pages, 2014.

[39] C. H. J. da Pinheiro, J. C. F. de Queiroz, L. Guimarães-Ferreira et al., "Local injections of adipose-derived mesenchymal stem cells modulate inflammation and increase angiogenesis ameliorating the dystrophic phenotype in dystrophin-deficient skeletal muscle," Stem Cell Reviews and Reports, vol. 8, no. 2, pp. 363374,2012

[40] Y. Tanabe, K. Esaki, and T. Nomura, "Skeletal muscle pathology in X chromosome-linked muscular dystrophy $(m d x)$ mouse," Acta Neuropathologica, vol. 69, no. 1-2, pp. 91-95, 1986.

[41] M. Hübscher, L. Vogt, T. Ziebart, and W. Banzer, "Immediate effects of acupuncture on strength performance: a randomized, controlled crossover trial," European Journal of Applied Physiology, vol. 110, no. 2, pp. 353-358, 2010.

[42] A. de Luca, B. Nico, J.-F. Rolland et al., "Gentamicin treatment in exercised $m d x$ mice: identification of dystrophin-sensitive pathways and evaluation of efficacy in work-loaded dystrophic muscle," Neurobiology of Disease, vol. 32, no. 2, pp. 243-253, 2008.

[43] R. Burdi, J.-F. Rolland, B. Fraysse et al., "Multiple pathological events in exercised dystrophic $\mathrm{mdx}$ mice are targeted by pentoxifylline: outcome of a large array of in vivo and ex vivo tests," Journal of Applied Physiology, vol. 106, no. 4, pp. 1311-1324, 2009.

[44] W. E. Hoffmann and P. F. Solter, "Diagnostic enzymology of domestic animals," in Clinical Biochemistry of Domestic Animals, J. Kaneko, Ed., chapter 12, pp. 351-378, Academic Press, San Diego, Calif, USA, 4th edition, 1989.

[45] J. G. Tidball and M. Wehling-Henricks, "The role of free radicals in the pathophysiology of muscular dystrophy," Journal of Applied Physiology, vol. 102, no. 4, pp. 1677-1686, 2007.

[46] H. Wang, Y. Pan, B. Xue et al., "The antioxidative effect of electro-acupuncture in a mouse model of Parkinson's disease," PLoS ONE, vol. 6, no. 5, Article ID e19790, 2011.

[47] C. Zhang, H.-Y. Feng, S.-L. Huang et al., “Therapy of Duchenne muscular dystrophy with umbilical cord blood stem cell transplantation," Chinese Journal of Medical Genetics, vol. 22, no. 4, pp. 399-405, 2005.

[48] N. Sych, M. Klunnik, O. Ivankova et al., "Efficacy of fetal stem cells in Duchenne muscular dystrophy therapy," Journal of Neurorestoratology, vol. 2, pp. 37-46, 2014.

[49] R. Kayali, J.-M. Ku, G. Khitrov, M. E. Jung, O. Prikhodko, and C. Bertoni, "Read-through compound 13 restores dystrophin expression and improves muscle function in the $M D X$ mouse model for duchenne muscular dystrophy," Human Molecular Genetics, vol. 21, no. 18, pp. 4007-4020, 2012. 


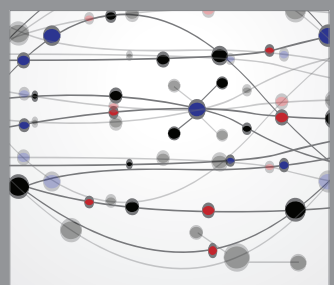

The Scientific World Journal
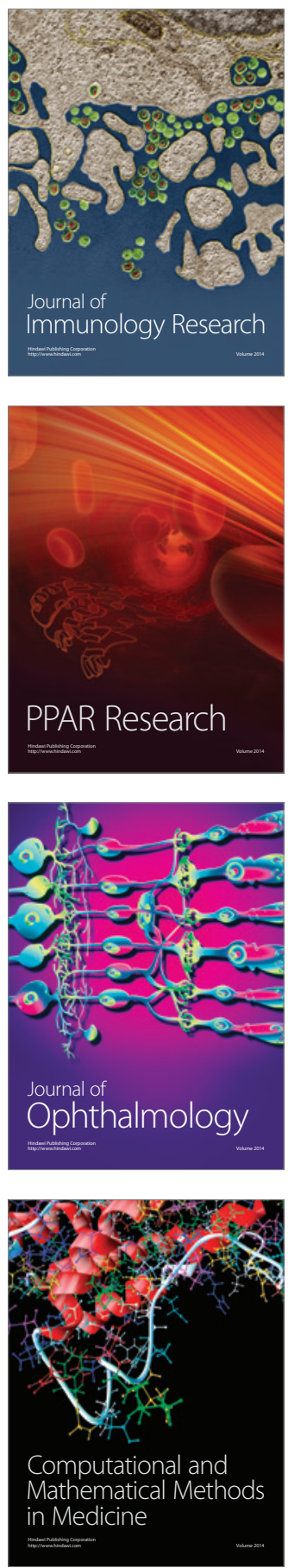

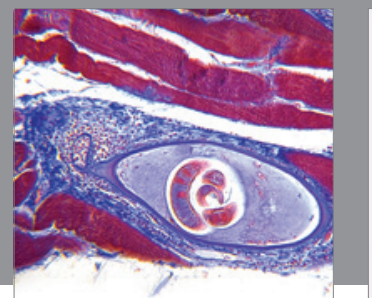

Gastroenterology

Research and Practice
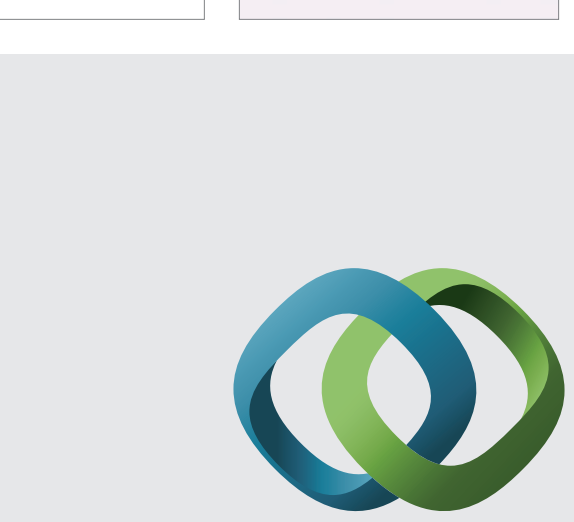

\section{Hindawi}

Submit your manuscripts at

http://www.hindawi.com
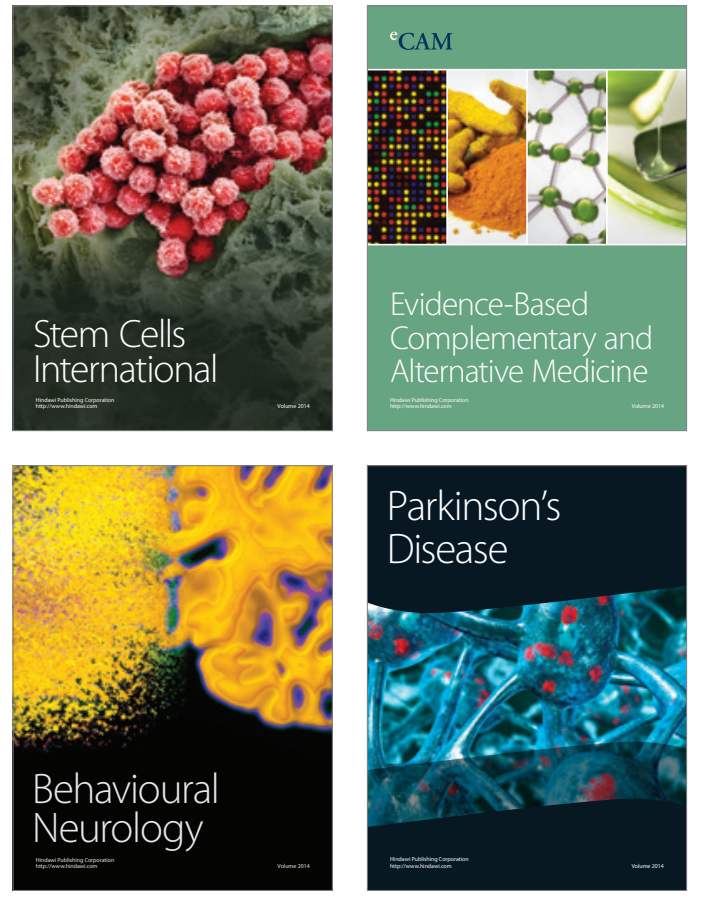
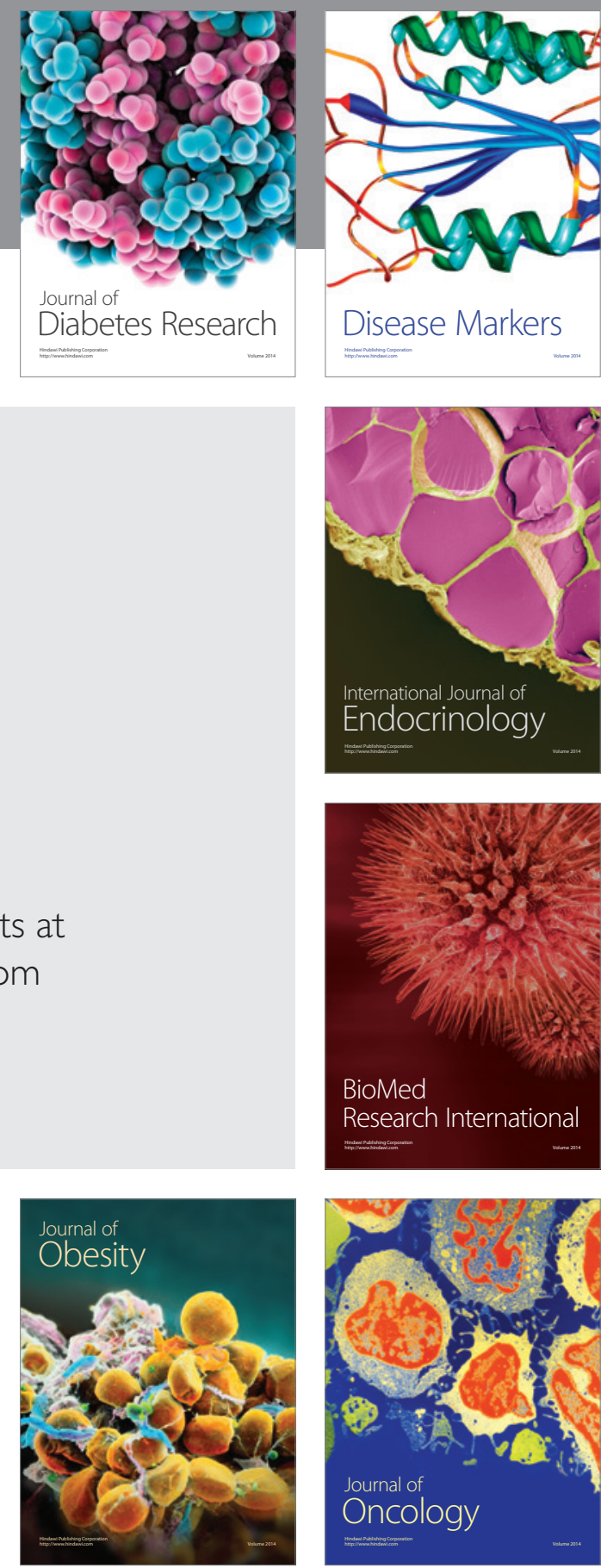

Disease Markers
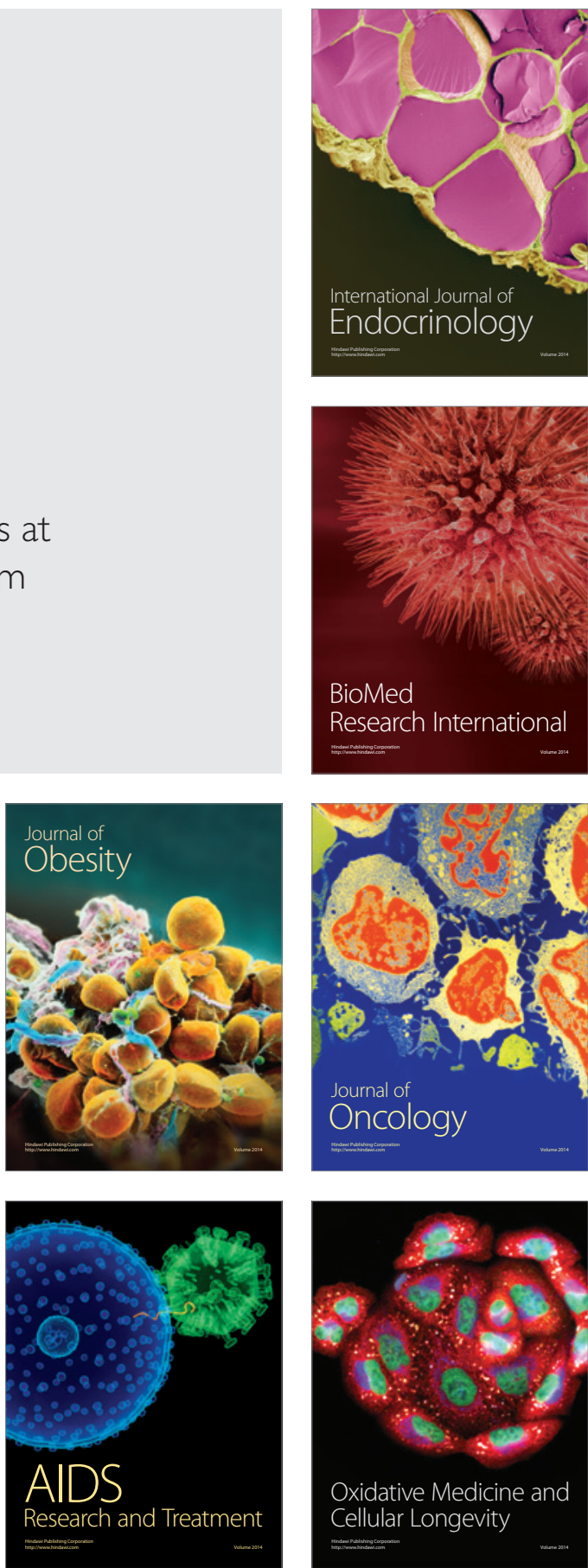